\title{
Minimum Energy Control of Fractional Positive Electrical Circuits with Bounded Inputs
}

\author{
Tadeusz Kaczorek ${ }^{1}$
}

Received: 24 April 2015 / Revised: 5 October 2015 / Accepted: 6 October 2015 /

Published online: 16 October 2015

(C) The Author(s) 2015. This article is published with open access at Springerlink.com

\begin{abstract}
Minimum energy control problem for the fractional positive electrical circuits with bounded inputs is formulated and solved. Sufficient conditions for the existence of solution to the problem are established. A procedure for solving of the problem is proposed and illustrated by example of fractional positive electrical circuit.
\end{abstract}

Keywords Fractional Positive - Electrical circuits - Minimum energy control · Bounded inputs · Procedure

\section{Introduction}

A dynamical system is called positive if its trajectory starting from any nonnegative initial state remains forever in the positive orthant for all nonnegative inputs. An overview of state of the art in positive theory is given in the monographs $[3,17]$. Variety of models having positive behavior can be found in engineering, economics, social sciences, biology and medicine, etc.

Mathematical fundamentals of the fractional calculus are given in the monographs [27-29]. The positive fractional linear systems have been investigated in $[5,6,8,18$, 19,21]. Stability of fractional linear continuous-time systems has been investigated in the papers $[1,20,21]$. The notion of practical stability of positive fractional linear systems has been introduced in [20]. Some recent interesting results in fractional systems theory and its applications can be found in [2,29,30,33,34].

Tadeusz Kaczorek

kaczorek@isep.pw.edu.pl

1 Faculty of Electrical Engineering, Bialystok University of Technology, Wiejska 45D,

15-351 Bialystok, Poland 
The minimum energy control problem for standard linear systems has been formulated and solved by J. Klamka in [22-25] and for 2D linear systems with variable coefficients in [22]. The controllability and minimum energy control problem of fractional discrete-time linear systems has been investigated by Klamka in [26]. The minimum energy control of fractional positive continuous-time linear systems has been addressed in $[4,11,13]$ and for descriptor positive discrete-time linear systems in $[4,6,10,12]$. The minimum energy control problem for positive and positive fractional electrical circuits has been investigated in [13-15].

In this paper, the minimum energy control problem for fractional positive electrical circuits with bounded inputs will be formulated and solved.

The paper is organized as follows. In Sect. 2, the basic definitions and theorems of the fractional positive electrical circuits are recalled and the necessary and sufficient conditions for the reachability of the electrical circuits are given.

The main result of the paper is given in Sect. 3 where minimum energy control problem is formulated, sufficient conditions for its solution are established, and a procedure is proposed. Illustrating example of fractional positive electrical circuit is given in Sect. 4. An extension to the method is presented in Sect. 5. Concluding remarks are given in Sect. 6.

The following notation will be used: $\mathfrak{R}$ - the set of real numbers, $\mathfrak{R}^{n \times m}$ — the set of $n \times m$ real matrices, $\Re_{+}^{n \times m}$ - the set of $n \times m$ matrices with nonnegative entries and $\Re_{+}^{n}=\Re_{+}^{n \times 1}, M_{n}$ - the set of $n \times n$ Metzler matrices (real matrices with nonnegative off-diagonal entries), $I_{n}$ - the $n \times n$ identity matrix.

\section{Preliminaries}

The following Caputo definition of the fractional derivative will be used [21]

$$
D^{\alpha} f(t)=\frac{\mathrm{d}^{\alpha}}{\mathrm{d} t^{\alpha}} f(t)=\frac{1}{\Gamma(n-\alpha)} \int_{0}^{t} \frac{f^{(n)}}{(t-\tau)^{\alpha+1-n}} \mathrm{~d} \tau, \quad n-1<\alpha \leq n \in N=\{1,2, \ldots\}
$$

where $\alpha \in \mathfrak{R}$ is the order of fractional derivative and $f^{(n)}(\tau)=\frac{\mathrm{d}^{n} f(\tau)}{\mathrm{d} \tau^{n}}$ and $\Gamma(x)=$ $\int_{0}^{\infty} \mathrm{e}^{-t} t^{x-1} \mathrm{~d} t$ is the gamma function.

Consider a fractional electrical circuit composed of resistors, coils, condensators and voltage (current) sources. Using the Kirchhoff's laws, we may describe the transient states in the electrical circuit by state equations $[7,9,16,17,21,28]$

$$
D^{\alpha} x(t)=A x(t)+B u(t), \quad 0<\alpha \leq 1
$$

where $x(t) \in \Re^{n}, u(t) \in \Re^{m}$ are the state and input vectors and $A \in \Re^{n \times n}, B \in \Re^{n \times m}$. 
Theorem 2.1 [21] The solution of Eq. (2.2) is given by

$$
x(t)=\Phi_{0}(t) x_{0}+\int_{0}^{t} \Phi(t-\tau) B u(\tau) \mathrm{d} \tau, \quad x(0)=x_{0}
$$

where

$$
\begin{aligned}
\Phi_{0}(t) & =E_{\alpha}\left(A t^{\alpha}\right)=\sum_{k=0}^{\infty} \frac{A^{k} t^{k \alpha}}{\Gamma(k \alpha+1)} \\
\Phi(t) & =\sum_{k=0}^{\infty} \frac{A^{k} t^{(k+1) \alpha-1}}{\Gamma[(k+1) \alpha]}
\end{aligned}
$$

and $E_{\alpha}\left(A t^{\alpha}\right)$ is the Mittag-Leffler matrix function [21].

Definition 2.1 [21] The fractional system (2.2) is called the (internally) positive fractional system if and only if $x(t) \in \Re_{+}^{n}, t \geq 0$ for any initial conditions $x_{0} \in \Re_{+}^{n}$ and all inputs $u(t) \in \Re_{+}^{m}, \quad t \geq 0$.

Theorem 2.2 [21] The continuous-time fractional system (2.2) is (internally) positive if and only if

$$
A \in M_{n}, \quad B \in \Re_{+}^{n \times m} .
$$

Definition 2.2 The state $x_{f} \in \Re_{+}^{n}$ of the fractional system (2.2) is called reachable in time $t_{f}$ if there exist an input $u(t) \in \Re_{+}^{m}, \quad t \in\left[0, t_{f}\right]$ which steers the state of system (2.2) from zero initial state $x_{0}=0$ to the state $x_{f}$.

A real square matrix is called monomial if each of its row and each of its column contain only one positive entry and the remaining entries are zero.

Theorem 2.3 The positive fractional system (2.2) is reachable in time $t \in\left[0, t_{f}\right]$ if and only if the matrix $A \in M_{n}$ is diagonal and the matrix $B \in \mathfrak{R}_{+}^{n \times n}$ is monomial.

Proof Sufficiency It is well known [17,21] that if $A \in M_{n}$ is diagonal, then $\Phi(t) \in$ $\Re_{+}^{n \times n}$ is also diagonal and if $B \in \Re_{+}^{n \times m}$ is monomial, then $B B^{\mathrm{T}} \in \Re_{+}^{n \times n}$ is also monomial. In this case, the matrix

$$
R_{f}=\int_{0}^{t_{f}} \Phi(\tau) B B^{\mathrm{T}} \Phi^{\mathrm{T}}(\tau) \mathrm{d} \tau \in \mathfrak{R}_{+}^{n \times n}
$$

is also monomial and $R_{f}^{-1} \in \mathfrak{R}_{+}^{n \times n}$. The input

$$
u(t)=B^{\mathrm{T}} \Phi^{\mathrm{T}}\left(t_{f}-t\right) R_{f}^{-1} x_{f}
$$


steers the state of the system (2.2) from $x_{0}=0$ to $x_{f}$ since using (2.3) for $x_{0}=0$ and (2.5) we obtain

$$
\begin{aligned}
x\left(t_{f}\right) & =\int_{0}^{t_{f}} \Phi\left(t_{f}-\tau\right) B u(\tau) \mathrm{d} \tau=\int_{0}^{t_{f}} \Phi\left(t_{f}-\tau\right) B B^{\mathrm{T}} \Phi^{\mathrm{T}}\left(t_{f}-\tau\right) \mathrm{d} \tau R_{f}^{-1} x_{f} \\
& =\int_{0}^{t_{f}} \Phi(\tau) B B^{\mathrm{T}} \Phi^{\mathrm{T}}(\tau) \mathrm{d} \tau R_{f}^{-1} x_{f}=x_{f} .
\end{aligned}
$$

The proof of necessity is given in [11].

\section{Problem Formulation and Its Solution}

Consider the fractional positive electrical circuit (2.2) with $A \in M_{n}$ and $B \in \Re_{+}^{n \times m}$ monomial. If the system is reachable in time $t \in\left[0, t_{f}\right]$, then usually there exist many different inputs $u(t) \in \Re_{+}^{n}$ that steers the state of the system from $x_{0}=0$ to $x_{f} \in \Re_{+}^{n}$. Among these inputs, we are looking for input $u(t) \in \mathfrak{R}_{+}^{n}, \quad t \in\left[0, t_{f}\right]$ satisfying the condition

$$
u(t) \leq U \in \mathfrak{R}_{+}^{n}, \quad t \in\left[0, t_{f}\right]
$$

that minimizes the performance index

$$
I(u)=\int_{0}^{t_{f}} u^{\mathrm{T}}(\tau) Q u(\tau) \mathrm{d} \tau
$$

where $Q \in \mathfrak{R}_{+}^{n \times n}$ is a symmetric positive defined matrix and $Q^{-1} \in \Re_{+}^{n \times n}$.

The performance index (3.2) is a measure of the energy used for steering the state of the systems from $x_{0}=0$ to $x_{f}$.

The minimum energy control problem for the fractional positive electrical circuit (2.2) can be stated as follows.

Given the matrices $A \in M_{n}, B \in \Re_{+}^{n \times m}, \alpha, U \in \Re_{+}^{n}$ and $Q \in \Re_{+}^{n \times n}$ of the performance matrix (3.2), $x_{f} \in \mathfrak{R}_{+}^{n}$ and $t>0$, find an input $u(t) \in \mathfrak{R}_{+}^{n}$ for $t \in\left[0, t_{f}\right]$ satisfying (3.1) that steers the state vector of the system from $x_{0}=0$ to $x_{f} \in \Re_{+}^{n}$ and minimizes the performance index (3.2).

To solve the problem, we define the matrix

$$
W\left(t_{f}\right)=\int_{0}^{t_{f}} \Phi\left(t_{f}-\tau\right) B Q^{-1} B^{\mathrm{T}} \Phi^{\mathrm{T}}\left(t_{f}-\tau\right) \mathrm{d} \tau
$$

where $\Phi(t)$ is defined by (2.5). From (3.3) and Theorem 2.3, it follows that the matrix (3.3) is monomial if and only if the fractional positive electrical circuit (2.2) is reachable in time $\left[0, t_{f}\right]$. In this case, we may define the input 


$$
\hat{u}(t)=Q^{-1} B^{\mathrm{T}} \Phi^{\mathrm{T}}\left(t_{f}-t\right) W^{-1}\left(t_{f}\right) x_{f} \quad \text { for } t \in\left[0, t_{f}\right] .
$$

Note that the input (3.4) satisfies the condition $u(t) \in \Re_{+}^{n}$ for $t \in\left[0, t_{f}\right]$ if

$$
Q^{-1} \in \mathfrak{R}_{+}^{n \times n} \text { and } W^{-1}\left(t_{f}\right) \in \Re_{+}^{n \times n} \text {. }
$$

Theorem 3.1 Let $\bar{u}(t) \in \Re_{+}^{n}$ for $t \in\left[0, t_{f}\right]$ be an input satisfying (3.1) that steers the state of the fractional positive electrical circuit (2.2) from $x_{0}=0$ to $x_{f} \in \Re_{+}^{n}$. Then the input (3.4) satisfying (3.1) also steers the state of the electrical circuit from $x_{0}=0$ to $x_{f} \in \Re_{+}^{n}$ and minimizes the performance index (3.2), i.e., $I(\hat{u}) \leq I(\bar{u})$.

The minimal value of the performance index (3.2) is equal to

$$
I(\hat{u})=x_{f}^{\mathrm{T}} W^{-1}\left(t_{f}\right) x_{f} .
$$

Proof If the conditions (3.5) are met, then the input (3.4) is well defined and $\hat{u}(t) \in \mathfrak{R}_{+}^{n}$ for $t \in\left[0, t_{f}\right]$. We shall show that the input steers the state of the electrical circuit from $x_{0}=0$ to $x_{f} \in \Re_{+}^{n}$. Substitution of (3.4) into (2.3) for $t=t_{f}$ and $x_{0}=0$ yields

$$
x\left(t_{f}\right)=\int_{0}^{t_{f}} \Phi\left(t_{f}-\tau\right) B \hat{u}(\tau) \mathrm{d} \tau=\int_{0}^{t_{f}} \Phi\left(t_{f}-\tau\right) B Q^{-1} B^{\mathrm{T}} \Phi^{\mathrm{T}}\left(t_{f}-\tau\right) \mathrm{d} \tau W_{f}^{-1} x_{f}=x_{f}
$$

since (3.3) holds. By assuming the inputs $\bar{u}(t)$ and $\hat{u}(t), t \in\left[0, t_{f}\right]$ steers the state of the system from $x_{0}=0$ to $x_{f} \in \mathfrak{R}_{+}^{n}$, i.e.,

$$
x_{f}=\int_{0}^{t_{f}} \Phi\left(t_{f}-\tau\right) B \bar{u}(\tau) \mathrm{d} \tau=\int_{0}^{t_{f}} \Phi\left(t_{f}-\tau\right) B \hat{u}(\tau) \mathrm{d} \tau
$$

and

$$
\int_{0}^{t_{f}} \Phi\left(t_{f}-\tau\right) B[\bar{u}(\tau)-\hat{u}(\tau)] \mathrm{d} \tau=0 .
$$

By transposition of (3.7c) and postmultiplication by $W^{-1}\left(t_{f}\right) x_{f}$, we obtain

$$
\int_{0}^{t_{f}}[\bar{u}(\tau)-\hat{u}(\tau)]^{\mathrm{T}} B^{\mathrm{T}} \Phi^{\mathrm{T}}\left(t_{f}-\tau\right) \mathrm{d} \tau W^{-1}\left(t_{f}\right) x_{f}=0 .
$$

Substitution of (3.4) into (3.8) yields

$$
\int_{0}^{t_{f}}[\bar{u}(\tau)-\hat{u}(\tau)]^{\mathrm{T}} B^{\mathrm{T}} \Phi^{\mathrm{T}}\left(t_{f}-\tau\right) \mathrm{d} \tau W^{-1} x_{f}=\int_{0}^{t_{f}}[\bar{u}(\tau)-\hat{u}(\tau)]^{\mathrm{T}} Q \hat{u}(\tau) \mathrm{d} \tau=0 .
$$


Using (3.9), it is easy to verify that

$$
\int_{0}^{t_{f}} \bar{u}(\tau)^{\mathrm{T}} Q \bar{u}(\tau) \mathrm{d} \tau=\int_{0}^{t_{f}} \hat{u}(\tau)^{\mathrm{T}} Q \hat{u}(\tau) \mathrm{d} \tau+\int_{0}^{t_{f}}[\bar{u}(\tau)-\hat{u}(\tau)]^{\mathrm{T}} Q[\bar{u}(\tau)-\hat{u}(\tau)] \mathrm{d} \tau .
$$

From (3.10), it follows that $I(\hat{u})<I(\bar{u})$ since the second term in the right-hand side of the inequality is nonnegative. To find the minimal value of the performance index (3.2), we substitute (3.4) into (3.2) and we obtain

$$
\begin{aligned}
I(\hat{u}) & =\int_{0}^{t_{f}} \hat{u}^{\mathrm{T}}(\tau) Q u(\hat{u}) \mathrm{d} \tau=x_{f}^{\mathrm{T}} W^{-1} \int_{0}^{t_{f}} \Phi\left(t_{f}-\tau\right) B Q^{-1} B^{\mathrm{T}} \Phi^{\mathrm{T}}\left(t_{f}-\tau\right) \mathrm{d} \tau W^{-1} x_{f} \\
& =x_{f}^{\mathrm{T}} W^{-1} x_{f}
\end{aligned}
$$

since (3.3) holds.

\section{Procedure and Example}

To find $t \in\left[0, t_{f}\right]$ for which $\hat{u}(t) \in \Re_{+}^{n}$ reaches its minimal value using (3.4), we compute the derivative

$$
\frac{\mathrm{d} \hat{u}(t)}{\mathrm{d} t}=Q^{-1} B^{\mathrm{T}} \Psi(t) W^{-1}\left(t_{f}\right) x_{f}, \quad t \in\left[0, t_{f}\right]
$$

where

$$
\Psi(t)=\frac{\mathrm{d}}{\mathrm{d} t}\left[\Phi^{\mathrm{T}}\left(t_{f}-t\right)\right] .
$$

Knowing $\Psi(t)$ and using the equality

$$
\Psi(t) W^{-1}\left(t_{f}\right) x_{f}=0
$$

we can find $t \in\left[0, t_{f}\right]$ for which $\hat{u}(t)$ reaches its maximal value.

Note that if the electrical circuit is asymptotically stable $\lim _{t \rightarrow \infty} \Phi(t)=0$, then $\hat{u}(t)$ reaches its maximal value for $t=t_{f}$ and if it is unstable, then for $t=0$.

From the above considerations, we have the following procedure for computation of the optimal inputs satisfying the condition (3.1) that steers the state of the system from $x_{0}=0$ to $x_{f} \in \mathfrak{R}_{+}^{n}$ and minimizes the performance index (3.2).

Procedure 4.1 Step 1 Knowing $A \in M_{n}$ and using (2.5), compute $\Phi(t)$.

Step 2 Using (3.3), compute the matrix $W_{f}$ for given $A, B, Q, \alpha$ and some $t_{f}$.

Step 3 Using (3.4) and (4.3), find $t_{f}$ for which $\hat{u}(t)$ satisfying (3.1) reaches its maximal value and the desired $\hat{u}(t)$ for given $U \in \mathfrak{R}_{+}^{n}$ and $x_{f} \in \mathfrak{R}_{+}^{n}$.

Step 4 Using (3.6), compute the maximal value of the performance index. 
Fig. 1 Electrical circuit

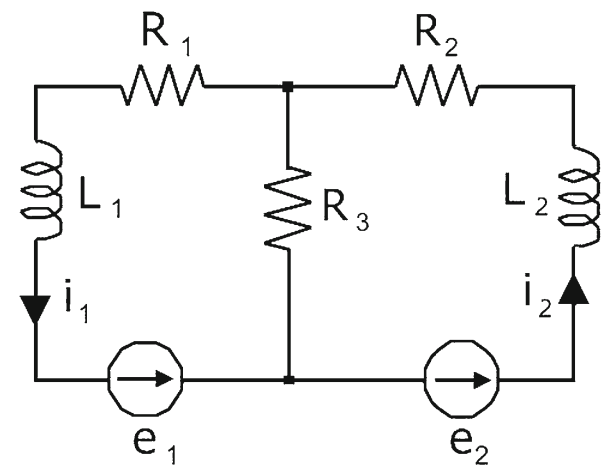

Example 4.1 Following [17], consider the fractional electrical circuit shown in Fig. 1 with given resistances $R_{1}, R_{2}, R_{3}$, inductances $L_{1}, L_{2}$ and source voltages $e_{1}, e_{2}$.

Using the Kirchhoff's laws, we can write the equations

$$
\begin{aligned}
& e_{1}=R_{3}\left(i_{1}-i_{2}\right)+R_{1} i_{1}+L_{1} \frac{\mathrm{d}^{\alpha} i_{1}}{\mathrm{~d} t^{\alpha}}, \\
& e_{2}=R_{3}\left(i_{2}-i_{1}\right)+R_{2} i_{2}+L_{2} \frac{\mathrm{d}^{\alpha} i_{2}}{\mathrm{~d} t^{\alpha}}
\end{aligned}
$$

where $0<\alpha<1$, which can be written in the form

$$
\frac{\mathrm{d}^{\alpha}}{\mathrm{d} t^{\alpha}}\left[\begin{array}{l}
i_{1} \\
i_{2}
\end{array}\right]=A\left[\begin{array}{l}
i_{1} \\
i_{2}
\end{array}\right]+B\left[\begin{array}{l}
e_{1} \\
e_{2}
\end{array}\right]
$$

where

$$
A=\left[\begin{array}{cc}
-\frac{R_{1}+R_{3}}{L_{1}} & \frac{R_{3}}{L_{1}} \\
\frac{R_{3}}{L_{2}} & -\frac{R_{2}+R_{3}}{L_{2}}
\end{array}\right], \quad B=\left[\begin{array}{cc}
\frac{1}{L_{1}} & 0 \\
0 & \frac{1}{L_{2}}
\end{array}\right] .
$$

The fractional electrical circuit is positive since the matrix $A$ is a Metzler matrix and the matrix $B$ has nonnegative entries.

In [17], it was shown that the electrical circuit is reachable if $R_{3}=0$. In this case, the matrix $A$ has the form

$$
A=\left[\begin{array}{cc}
-\frac{R_{1}}{L_{1}} & 0 \\
0 & -\frac{R_{2}}{L_{2}}
\end{array}\right]
$$

Consider the fractional positive reachable electrical circuit shown in Fig. 1 for $R_{1}=$ $R_{2}=1, \quad R_{3}=0, L_{1}=L_{2}=1$ and $\alpha=0.7$. Compute the input $\hat{u}(t)$ satisfying the condition

$$
\hat{u}(t)=\left[\begin{array}{l}
e_{1} \\
e_{2}
\end{array}\right] \leq\left[\begin{array}{l}
20 \\
20
\end{array}\right] \text { for } t \in\left[0, t_{f}\right]
$$


that steers the state of the electrical circuit from zero state to final state $x_{f}=\left[\begin{array}{ll}1 & 1\end{array}\right]^{\mathrm{T}}$ ( $T$ denotes the transpose) and minimizes the performance index (3.1) with

$$
Q=\left[\begin{array}{ll}
2 & 0 \\
0 & 2
\end{array}\right]
$$

Using the Procedure 4.1, we obtain the following:

Step 1 Taking into account that

$$
A=\left[\begin{array}{cc}
-1 & 0 \\
0 & -1
\end{array}\right]
$$

and using (2.5), we obtain

$$
\Phi(t)=\sum_{k=0}^{\infty} \frac{A^{k} t^{(k+1) \alpha-1}}{\Gamma[(k+1) \alpha]}=\sum_{k=0}^{\infty}\left[\begin{array}{cc}
(-1)^{k} & 0 \\
0 & (-1)^{k}
\end{array}\right] \frac{t^{(k+1) 0.7-1}}{\Gamma[(k+1) 0.7]}
$$

Step 2 Using (3.3), (4.8), (4.9) and taking into account that $B=I_{2}$, we get

$$
\begin{aligned}
& W\left(t_{f}\right)=\int_{0}^{t_{f}} \Phi\left(t_{f}-\tau\right) B Q^{-1} B^{\mathrm{T}} \Phi^{\mathrm{T}}\left(t_{f}-\tau\right) \mathrm{d} \tau=\int_{0}^{t_{f}} \Phi(\tau) B Q^{-1} B^{\mathrm{T}} \Phi^{\mathrm{T}}(\tau) \mathrm{d} \tau \\
& =\frac{1}{2} \int_{0}^{t_{f}} \Phi^{2}(\tau) \mathrm{d} \tau=\frac{1}{2} \int_{0}^{t_{f}}\left[\sum_{k=0}^{\infty}\left[\begin{array}{cc}
(-1)^{k} & 0 \\
0 & (-1)^{k}
\end{array}\right] \frac{t^{(k+1) 0.7-1}}{\Gamma[(k+1) 0.7]}\right]^{2} \mathrm{~d} \tau \\
& =\frac{1}{2} \int_{0}^{t_{f}} \sum_{k=0}^{\infty} \sum_{l=0}^{\infty}\left[\begin{array}{cc}
(-1)^{k+l} & 0 \\
0 & (-1)^{k+l}
\end{array}\right] \frac{\tau^{(k+l) 0.7-0.6}}{\Gamma[(k+1) 0.7] \Gamma[(l+1) 0.7]} \mathrm{d} \tau \\
& =\frac{1}{2} \sum_{k=0}^{\infty} \sum_{l=0}^{\infty}\left[\begin{array}{cc}
(-1)^{k+l} & 0 \\
0 & (-1)^{k+l}
\end{array}\right] \frac{1}{\Gamma[(k+1) 0.7] \Gamma[(l+1) 0.7]} \frac{t_{f}^{(k+l) 0.7+0.4}}{(k+l) 0.7+0.4} \\
& =\left[\begin{array}{cc}
0.3729 & 0 \\
0 & 0.3729
\end{array}\right] \text {. }
\end{aligned}
$$

Step 3 Using (2.7) and (2.8), we obtain

$$
\begin{aligned}
\hat{u}(t) & =Q^{-1} B^{\mathrm{T}} \Phi^{\mathrm{T}}\left(t_{f}-t\right) W^{-1}\left(t_{f}\right) x_{f} \\
& =\frac{1}{2}\left(\sum_{k=0}^{\infty}\left[\begin{array}{cc}
(-1)^{k} & 0 \\
0 & (-1)^{k}
\end{array}\right] \frac{\left(t_{f}-t\right)^{(k+1) 0.7-1}}{\Gamma[(k+1) 0.7]}\right) W^{-1}\left(t_{f}\right)\left[\begin{array}{l}
1 \\
1
\end{array}\right]
\end{aligned}
$$

Note that the electrical circuit is stable. Therefore, $\hat{u}(t)$ reach its maximal value for $t=t_{f}$ (Fig. 2). 

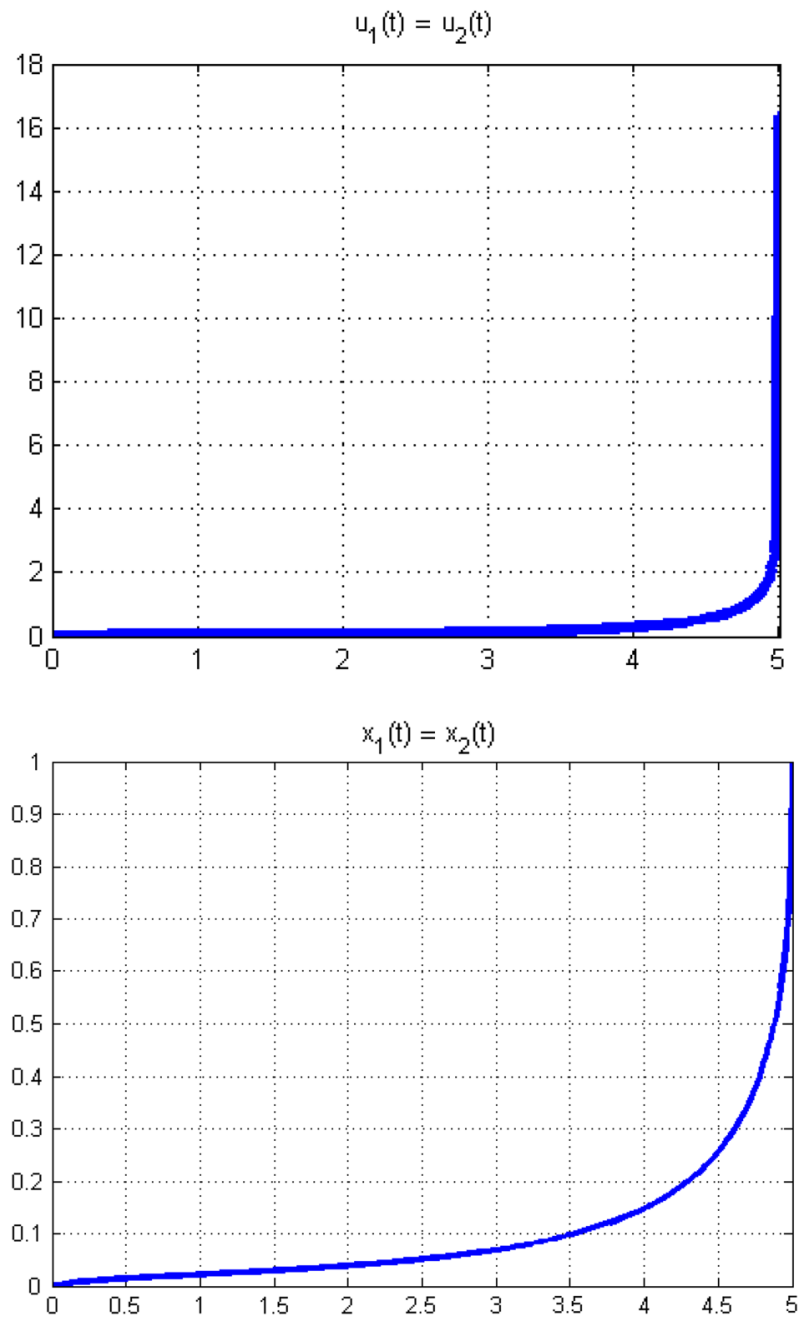

Fig. 2 Input signal and state variable for $t_{f}=5[\mathrm{~s}]$

Step 4 From (3.6), we have the minimal value of the performance index

$$
I(\hat{u})=x_{f}^{\mathrm{T}} W^{-1}\left(t_{f}\right) x_{f}
$$

where $W\left(t_{f}\right)$ is given by (4.11).

\section{Extension to Fractional Positive Electrical Circuits with Different Orders}

Consider an electrical circuit composed of resistors, $n$ capacitors and $m$ voltage (current) sources. Using the Kirchhoff's laws, we may describe the transient states in the electrical circuit by the fractional differential equation 


$$
\frac{\mathrm{d}^{\alpha} x(t)}{\mathrm{d} t^{\alpha}}=A x(t)+B u(t), \quad 0<\alpha<1
$$

where $x(t) \in \Re^{n}, u(t) \in \Re^{m}, A \in \Re^{n \times n}, B \in \Re^{n \times m}$. The components of the state vector $x(t)$ and input vector $u(t)$ are the voltages on the capacitors and source voltages, respectively. Similarly, using the Kirchhoff's laws, we may describe the transient states in the electrical circuit composed of resistances, inductances and voltage (current) sources by the fractional differential equation

$$
\frac{\mathrm{d}^{\beta} x(t)}{\mathrm{d} t^{\beta}}=A x(t)+B u(t), \quad 0<\beta<1
$$

where $x(t) \in \mathfrak{R}^{n}, u(t) \in \mathfrak{R}^{m}, A \in \mathfrak{R}^{n \times n}, B \in \mathfrak{R}^{n \times m}$. In this case, the components of the state vector $x(t)$ are the currents in the coils.

Now let us consider electrical circuit composed of resistors, capacitors, coils and voltage (current) sources. As the state variables (the components of the state vector $x(t))$, we choose the voltages on the capacitors and the currents in the coils. Using Eqs. (5.1), (5.2) and Kirchhoff's laws, we may write for the fractional linear circuits in the transient states the state equation

$$
\left[\begin{array}{c}
\frac{\mathrm{d}^{\alpha} x_{C}}{\mathrm{~d} t^{\alpha}} \\
\frac{\mathrm{d}^{\beta} x_{L}}{\mathrm{~d} t^{\beta}}
\end{array}\right]=\left[\begin{array}{ll}
A_{11} & A_{12} \\
A_{21} & A_{22}
\end{array}\right]\left[\begin{array}{l}
x_{C} \\
x_{L}
\end{array}\right]+\left[\begin{array}{l}
B_{1} \\
B_{2}
\end{array}\right] u, \quad 0<\alpha, \beta<1,
$$

where the components of $x_{C} \in \Re^{n_{1}}$ are voltages on the capacitors, the components of $x_{L} \in \Re^{n_{2}}$ are currents in the coils and the components of $u \in \Re^{m}$ are the source voltages and

$$
A_{i j} \in \Re^{n_{i} \times n_{j}}, \quad B_{i} \in \Re^{n_{i} \times m}, \quad i, j=1,2 .
$$

Theorem 5.1 The solution of Eq. (5.3) for $0<\alpha<1$; $0<\beta<1$ with initial conditions

$$
x_{C}(0)=x_{10} \text { and } x_{L}(0)=x_{20}
$$

has the form

$$
x(t)=\Phi_{0}(t) x_{0}+\int_{0}^{t}\left[\Phi_{1}(t-\tau) B_{10}+\Phi_{2}(t-\tau) B_{01}\right] u(\tau) \mathrm{d} \tau,
$$

where

$$
x(t)=\left[\begin{array}{l}
x_{1}(t) \\
x_{2}(t)
\end{array}\right], \quad x_{0}=\left[\begin{array}{l}
x_{10} \\
x_{20}
\end{array}\right], \quad B_{10}=\left[\begin{array}{c}
B_{1} \\
0
\end{array}\right], \quad B_{01}=\left[\begin{array}{c}
0 \\
B_{2}
\end{array}\right],
$$


Fig. 3 Fractional electrical circuit

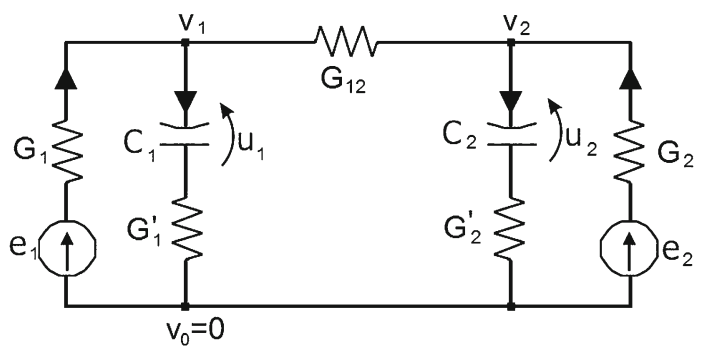

$$
\begin{aligned}
& T_{k l}=\left\{\begin{array}{l}
I_{n} \text { for } k=l=0 \\
{\left[\begin{array}{cc}
A_{11} & A_{12} \\
0 & 0
\end{array}\right] \text { for } k=1, l=0} \\
{\left[\begin{array}{cc}
0 & 0 \\
A_{21} & A_{22}
\end{array}\right] \text { for } k=0, l=1} \\
T_{10} T_{k-1, l}+T_{01} T_{k, l-1} \text { for } k+l>0
\end{array}\right. \\
& \Phi_{0}(t)=\sum_{k=0}^{\infty} \sum_{l=0}^{\infty} T_{k l} \frac{t^{k \alpha+l \beta}}{\Gamma(k \alpha+l \beta+1)}, \\
& \Phi_{1}(t)=\sum_{k=0}^{\infty} \sum_{l=0}^{\infty} T_{k l} \frac{t^{(k+1) \alpha+l \beta-1}}{\Gamma[(k+1) \alpha+l \beta]}, \\
& \Phi_{2}(t)= \sum_{k=0}^{\infty} \sum_{l=0}^{\infty} T_{k l} \frac{t^{k \alpha+(l+1) \beta-1}}{\Gamma[k \alpha+(l+1) \beta]} .
\end{aligned}
$$

Proof is given in [18,21].

The extension of Theorem 5.1 to systems consisting of $n$ subsystems with different fractional orders is given in [18,21].

Example 5.1 Consider the fractional electrical circuit shown in Fig. 3 [19,31,32] with given source voltages $e_{1}, e_{2}$, ultracapacitor $C_{1}=1$ of the fractional order $\alpha=0.7$, ultracapacitor $C_{2}=2$ of the fractional order $\beta=0.6$, conductances $G_{1}=4, G_{1}^{\prime}=$ $4, G_{2}=3, G_{2}^{\prime}=6, G_{12}=0$ and $N=n_{1}+n_{2}=2$.

Using the Kirchhoff's laws, we can write the equations

$$
\begin{aligned}
& C_{1} \frac{\mathrm{d}^{\alpha} u_{1}}{\mathrm{~d} t^{\alpha}}=G_{1}^{\prime}\left(v_{1}-u_{1}\right), \\
& C_{2} \frac{\mathrm{d}^{\beta} u_{2}}{\mathrm{~d} t^{\beta}}=G_{2}^{\prime}\left(v_{2}-u_{2}\right)
\end{aligned}
$$

and

$$
\left[\begin{array}{cc}
\left(G_{1}+G_{1}^{\prime}\right) & 0 \\
0 & \left(G_{2}+G_{2}^{\prime}\right)
\end{array}\right]\left[\begin{array}{l}
v_{1} \\
v_{2}
\end{array}\right]=\left[\begin{array}{cc}
G_{1}^{\prime} & 0 \\
0 & G_{2}^{\prime}
\end{array}\right]\left[\begin{array}{l}
u_{1} \\
u_{2}
\end{array}\right]+\left[\begin{array}{cc}
G_{1} & 0 \\
0 & G_{2}
\end{array}\right]\left[\begin{array}{l}
e_{1} \\
e_{2}
\end{array}\right] .
$$


From (5.8), we obtain

$$
\begin{aligned}
{\left[\begin{array}{l}
v_{1} \\
v_{2}
\end{array}\right]=} & {\left[\begin{array}{cc}
\left(G_{1}+G_{1}^{\prime}\right) & 0 \\
0 & \left(G_{2}+G_{2}^{\prime}\right)
\end{array}\right]^{-1}\left[\begin{array}{cc}
G_{1}^{\prime} & 0 \\
0 & G_{2}^{\prime}
\end{array}\right]\left[\begin{array}{l}
u_{1} \\
u_{2}
\end{array}\right] } \\
& +\left[\begin{array}{cc}
\left(G_{1}+G_{1}^{\prime}\right) & 0 \\
0 & \left(G_{2}+G_{2}^{\prime}\right)
\end{array}\right]^{-1}\left[\begin{array}{cc}
G_{1} & 0 \\
0 & G_{2}
\end{array}\right]\left[\begin{array}{l}
e_{1} \\
e_{2}
\end{array}\right] .
\end{aligned}
$$

Substitution of (5.9) into

$$
\left[\begin{array}{c}
\frac{\mathrm{d}^{\alpha} u_{1}}{\mathrm{~d} t^{\alpha}} \\
\frac{\mathrm{d}^{\beta} u_{2}}{\mathrm{~d} t^{\beta}}
\end{array}\right]=\left[\begin{array}{cc}
-\frac{G_{1}^{\prime}}{C_{1}} & 0 \\
0 & -\frac{G_{2}^{\prime}}{C_{2}}
\end{array}\right]\left[\begin{array}{l}
u_{1} \\
u_{2}
\end{array}\right]+\left[\begin{array}{cc}
\frac{G_{1}^{\prime}}{C_{1}} & 0 \\
0 & \frac{G_{2}^{\prime}}{C_{2}}
\end{array}\right]\left[\begin{array}{l}
v_{1} \\
v_{2}
\end{array}\right]
$$

we obtain

$$
\left[\begin{array}{c}
\frac{\mathrm{d}^{\alpha} u_{1}}{\mathrm{~d} t^{\alpha}} \\
\frac{\mathrm{d}^{\beta} u_{2}}{\mathrm{~d} t^{\beta}}
\end{array}\right]=A\left[\begin{array}{l}
u_{1} \\
u_{2}
\end{array}\right]+B\left[\begin{array}{l}
e_{1} \\
e_{2}
\end{array}\right]
$$

where

$$
\begin{aligned}
& A=\left[\begin{array}{cc}
\frac{G_{1}^{\prime}}{C_{1}}\left(\frac{G_{1}^{\prime}}{\left(G_{1}+G_{1}^{\prime}\right)}-1\right) & 0 \\
0 & \frac{G_{2}^{\prime}}{C_{2}}\left(\frac{G_{2}^{\prime}}{\left(G_{2}+G_{2}^{\prime}\right)}-1\right)
\end{array}\right]=\left[\begin{array}{cc}
-2 & 0 \\
0 & -1
\end{array}\right]=\left[\begin{array}{cc}
A_{1} & 0 \\
0 & A_{2}
\end{array}\right], \\
& B=\left[\begin{array}{cc}
\frac{G_{1}^{\prime} G_{1}}{C_{1}\left(G_{1}+G_{1}^{\prime}\right)} & 0 \\
0 & \frac{G_{2}^{\prime} G_{2}}{C_{2}\left(G_{2}+G_{2}^{\prime}\right)}
\end{array}\right]=\left[\begin{array}{ll}
2 & 0 \\
0 & 1
\end{array}\right]=\left[\begin{array}{l}
B_{1} \\
B_{2}
\end{array}\right] .
\end{aligned}
$$

From (5.12), it follows that $A$ is a diagonal Metzler matrix and the matrix $B$ is monomial matrix with positive diagonal entries. Therefore, the fractional electrical circuit is positive for all values of the conductances and capacitances.

Find the optimal input (source voltage) $\hat{e}(t) \in \Re_{+}^{2}, \quad t \in\left[0, t_{f}\right]$ satisfying the condition

$$
\hat{e}(t)=\left[\begin{array}{l}
\hat{e}_{1}(t) \\
\hat{e}_{2}(t)
\end{array}\right]<\left[\begin{array}{l}
1 \\
1
\end{array}\right] \text { for } t \in\left[0, t_{f}\right]
$$

for the performance index (3.2) with $Q=\operatorname{diag}[2,2]$ which steers the system from initial state (voltage drop on capacitances) $u_{0}=\left[\begin{array}{ll}0 & 0\end{array}\right]^{\mathrm{T}}$ to the finite state $u_{f}=\left[\begin{array}{ll}2 & 3\end{array}\right]^{\mathrm{T}}$ and minimizes the performance index (3.2) with (5.12).

Using (5.6) and (5.12), we obtain

$$
\Phi_{1}(t) B_{10}+\Phi_{2}(t) B_{01}=\left[\begin{array}{cc}
\Phi_{11}^{1}(t) & 0 \\
0 & \Phi_{22}^{2}(t)
\end{array}\right]\left[\begin{array}{l}
B_{1} \\
B_{2}
\end{array}\right]=M(t)
$$


where

$$
\begin{aligned}
& \Phi_{11}^{1}(t)=\sum_{k=0}^{\infty} \sum_{l=0}^{\infty} \frac{t^{(k+1) 0.7+l 0.6-1}}{\Gamma[(k+1) 0.7+l 0.6]}(-2)^{k}, \\
& \Phi_{22}^{2}(t)=\sum_{k=0}^{\infty} \sum_{l=0}^{\infty} \frac{t^{k 0.7+(l+1) 0.6-1}}{\Gamma[k 0.7+(l+1) 0.6]}(-1)^{l} .
\end{aligned}
$$

From (5.6a), (5.12) and (3.3), we have

$$
\begin{aligned}
W\left(t_{f}\right) & =\int_{0}^{t_{f}} M\left(t_{f}-\tau\right) Q^{-1} M^{\mathrm{T}}\left(t_{f}-\tau\right) \mathrm{d} \tau \\
& =\int_{0}^{t_{f}}\left[\begin{array}{cc}
\Phi_{11}^{1}(\tau) & 0 \\
0 & \Phi_{22}^{2}(\tau)
\end{array}\right]\left[\begin{array}{ll}
8 & 0 \\
0 & 2
\end{array}\right]\left[\begin{array}{cc}
\Phi_{11}^{1}(\tau) & 0 \\
0 & \Phi_{22}^{2}(\tau)
\end{array}\right]^{\mathrm{T}} \mathrm{d} \tau .
\end{aligned}
$$

Note that the electrical circuit is stable. Therefore, $\hat{e}(t)$ reach its maximal value for $t=t_{f}$.

Now using (3.4) and (5.16), we obtain

$$
\begin{aligned}
& \hat{e}(t)=Q^{-1} M^{\mathrm{T}}\left(t_{f}-t\right) W^{-1}\left(t_{f}\right) u_{f} \\
& =\left[\begin{array}{cc}
1 & 0 \\
0 & 0.5
\end{array}\right]\left[\begin{array}{cc}
\Phi_{11}^{1}\left(t_{f}-t\right) & 0 \\
0 & \Phi_{22}^{2}\left(t_{f}-t\right)
\end{array}\right]^{\mathrm{T}} W^{-1}\left(t_{f}\right)\left[\begin{array}{l}
2 \\
3
\end{array}\right]
\end{aligned}
$$

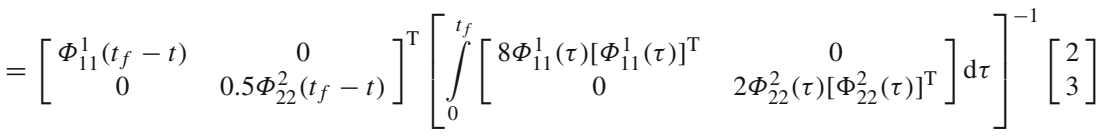

$$
\begin{aligned}
& =\left[\begin{array}{c}
0.25\left[\Phi_{11}^{1}\left(t_{f}-t\right)\right]^{\mathrm{T}} \int_{0}^{t_{f}}\left[\Phi_{11}^{1}(\tau)\left[\Phi_{11}^{1}(\tau)\right]^{\mathrm{T}}\right]^{-1} \mathrm{~d} \tau \\
1.5\left[\Phi_{22}^{2}\left(t_{f}-t\right)\right]^{\mathrm{T}} \int_{0}^{t_{f}}\left[\Phi_{22}^{2}(\tau)\left[\Phi_{22}^{2}(\tau)\right]^{\mathrm{T}}\right]^{-1} \mathrm{~d} \tau
\end{array}\right],
\end{aligned}
$$

The minimal value of $t_{f}$ satisfying the condition (3.1) can be found from the inequality

$$
\left[\begin{array}{l}
0.25\left[\Phi_{11}^{1}\left(t_{f}-t\right)\right]^{\mathrm{T}} \int_{0}^{t_{f}}\left[\Phi_{11}^{1}(\tau)\left[\Phi_{11}^{1}(\tau)\right]^{\mathrm{T}}\right]^{-1} \mathrm{~d} \tau \\
1.5\left[\Phi_{22}^{2}\left(t_{f}-t\right)\right]^{\mathrm{T}} \int_{0}^{t_{f}}\left[\Phi_{22}^{2}(\tau)\left[\Phi_{22}^{2}(\tau)\right]^{\mathrm{T}}\right]^{-1} \mathrm{~d} \tau
\end{array}\right]<\left[\begin{array}{l}
1 \\
1
\end{array}\right] \text { for } t \in\left[0, t_{f}\right]
$$

and the minimal value of the performance index (3.2) is equal to 


$$
\begin{aligned}
I(\hat{e}) & =u_{f}^{\mathrm{T}} W^{-1} u_{f}=\left[\begin{array}{ll}
2 & 3
\end{array}\right]\left[\int_{0}^{t_{f}}\left[\begin{array}{cc}
8 \Phi_{11}^{1}(\tau)\left[\Phi_{11}^{1}(\tau)\right]^{\mathrm{T}} & 0 \\
0 & 2 \Phi_{22}^{2}(\tau)\left[\Phi_{22}^{2}(\tau)\right]^{\mathrm{T}}
\end{array}\right] \mathrm{d} \tau\right]^{-1}\left[\begin{array}{l}
2 \\
3
\end{array}\right] \\
& =0.5 \int_{0}^{t_{f}}\left[\Phi_{11}^{1}(\tau)\left[\Phi_{11}^{1}(\tau)\right]^{\mathrm{T}}\right]^{-1} \mathrm{~d} \tau+3 \int_{0}^{t_{f}}\left[\Phi_{22}^{2}(\tau)\left[\Phi_{22}^{2}(\tau)\right]^{\mathrm{T}}\right]^{-1} \mathrm{~d} \tau .
\end{aligned}
$$

\section{Concluding Remarks}

Necessary and sufficient conditions for the reachability of the fractional positive electrical circuits have been established (Theorem 2.3). The minimum energy control problem for the fractional positive electrical circuits with bounded inputs has been formulated and solved. Sufficient conditions for the existence of a solution to the problem have been given (Theorem 3.1), and a procedure for computation of optimal input and the minimal value of performance index has been proposed. The effectiveness of the procedure has been demonstrated on the example of fractional positive electrical circuit. The presented method can be extended to positive discrete-time linear systems and to fractional positive discrete-time linear systems with bounded inputs.

Acknowledgments This work was supported under work S/WE/1/11.

Open Access This article is distributed under the terms of the Creative Commons Attribution 4.0 International License (http://creativecommons.org/licenses/by/4.0/), which permits unrestricted use, distribution, and reproduction in any medium, provided you give appropriate credit to the original author(s) and the source, provide a link to the Creative Commons license, and indicate if changes were made.

\section{References}

1. M. Busłowicz, Stability of linear continuous time fractional order systems with delays of the retarded type. Bull. Pol. Acad. Sci. Tech. 56(4), 319-324 (2008)

2. A. Dzieliński, D. Sierociuk, G. Sarwas, Ultracapacitor parameters identification based on fractional order model, in Proceedings of ECC'09, Budapest (2009)

3. L. Farina, S. Rinaldi, Positive Linear Systems: Theory and Applications (Wiley, New York, 2000)

4. T. Kaczorek, An extension of Klamka's method of minimum energy control to fractional positive discrete-time linear systems with bounded inputs. Bull. Pol. Acad. Sci. Tech. 62(2), 227-231 (2014)

5. T. Kaczorek, Asymptotic stability of positive fractional 2D linear systems. Bull. Pol. Acad. Sci. Tech. 57(3), 289-292 (2009)

6. T. Kaczorek, Checking of the positivity of descriptor linear systems by the use of the shuffle algorithm. Arch. Control Sci. 21(3), 287-298 (2011)

7. T. Kaczorek, Controllability and observability of linear electrical circuits. Electr. Rev. 87(9a), 248-254 (2011)

8. T. Kaczorek, Fractional positive continuous-time systems and their reachability. Int. J. Appl. Math. Comput. Sci. 18(2), 223-228 (2008)

9. T. Kaczorek, Linear Control Systems (Research Studies Press and Wiley, New York, 1992)

10. T. Kaczorek, Minimum energy control of descriptor positive discrete-time linear systems. COMPEL Int. J. Comput. Math. Electr. Electron. Eng. 33(3), 976-988 (2014)

11. T. Kaczorek, Minimum energy control of fractional positive continuous-time linear systems. Bull. Pol. Acad. Sci. Tech. 61(4), 803-807 (2013)

12. T. Kaczorek, Minimum energy control of fractional positive discrete-time linear systems with bounded inputs. Arch. Control Sci. 23(2), 205-211 (2013) 
13. T. Kaczorek, Minimum energy control of fractional positive electrical circuits, in Proceedings of 22th European Signal Processing Conference (2014)

14. T. Kaczorek, Minimum energy control of positive continuous-time linear systems with bounded inputs. Int. J. Appl. Math. Comput. Sci. 23(2), 725-730 (2013)

15. T. Kaczorek, Minimum energy control of positive electrical circuits, in Proceedings of 19th International Conference of Methods and Models in Automation and Robotics (2014)

16. T. Kaczorek, Polynomial approach to fractional descriptor electrical circuits, in Proceedings of 7th International Conference on Intelligent Information and Engineering Systems (2014)

17. T. Kaczorek, Positive $1 D$ and $2 D$ Systems (Springer, London, 2001)

18. T. Kaczorek, Positive linear systems consisting of $\mathrm{n}$ subsystems with different fractional orders. IEEE Trans. Circuits Syst. 58(6), 1203-1210 (2011)

19. T. Kaczorek, Positivity and reachability of fractional electrical circuits. Acta Mech. Autom. 5(2), 42-51 (2011)

20. T. Kaczorek, Practical stability of positive fractional discrete-time linear systems. Bull. Pol. Acad. Sci. Tech. 56(4), 313-317 (2008)

21. T. Kaczorek, Selected Problems of Fractional Systems Theory (Springer, Berlin, 2012)

22. T. Kaczorek, J. Klamka, Minimum energy control of $2 \mathrm{D}$ linear systems with variable coefficients. Int. J. Control 44(3), 645-650 (1986)

23. J. Klamka, Controllability and minimum energy control problem of fractional discrete-time systems, in New Trends in Nanotechnology and Fractional Calculus, ed. by D. Baleanu, Z.B. Guvenc, J.A. Tenreiro Machado (Springer, New York, 2010), pp. 503-509

24. J. Klamka, Controllability of Dynamical Systems (Kluwer Academic Press, Dordrecht, 1991)

25. J. Klamka, Minimum energy control of 2D systems in Hilbert spaces. Syst. Sci. 9(1-2), 33-42 (1983)

26. J. Klamka, Relative controllability and minimum energy control of linear systems with distributed delays in control. IEEE Trans. Autom. Control 21(4), 594-595 (1976)

27. K.B. Oldham, J. Spanier, The Fractional Calculus (Academic Press, New York, 1974)

28. P. Ostalczyk, Epitome of the Fractional Calculus: Theory and Its Applications in Automatics (Wydawnictwo Politechniki Łódzkiej, Łódź, 2008). (in Polish)

29. I. Podlubny, Fractional Differential Equations (Academic Press, San Diego, 1999)

30. A.G. Radwan, A.M. Soliman, A.S. Elwakil, A. Sedeek, On the stability of linear systems with fractionalorder elements. Chaos Solitons Fractals 40(5), 2317-2328 (2009)

31. Ł. Sajewski, Minimum energy control of fractional positive continuous-time linear systems with two different fractional orders and bounded inputs, in Advances in Modelling and Control of Non-integerOrder Systems, Lecture Notes in Electrical Engineering, vol. 320 (2015) pp. 171-181

32. Ł. Sajewski, Reachability, observability and minimum energy control of fractional positive continuoustime linear systems with two different fractional orders. Multidimens. Syst. Signal Process. (2014). doi:10.1007/s11045-014-0287-2

33. E.J. Solteiro Pires, J.A. Tenreiro Machado, P.B. Moura Oliveira, Fractional dynamics in genetic algorithms, in Workshop on Fractional Differentiation and Its Application vol. 2 (2006) pp. 414-419

34. B.M. Vinagre, C.A. Monje, A.J. Calderon, Fractional order systems and fractional order control actions, in Lecture 3 IEEE CDC'02 TW\#2: Fractional calculus Applications in Automatic Control and Robotics (2002) 